\title{
Eosinophilic esophagitis in adults: distinguishing features from gastroesophageal reflux disease: a study of 41 patients
}

\author{
Jeremy R Parfitt ${ }^{1}$, James C Gregor ${ }^{2}$, Neville G Suskin ${ }^{2}$, Hani A Jawa ${ }^{2}$ and David K Driman ${ }^{1}$ \\ ${ }^{1}$ Department of Pathology, London Health Sciences Centre and University of Western Ontario, London, \\ Ontario, Canada and ${ }^{2}$ Department of Medicine, London Health Sciences Centre and University of Western \\ Ontario, London, Ontario, Canada
}

\begin{abstract}
Eosinophilic esophagitis in adults is a recently described entity occurring in young males with dysphagia, in whom esophageal biopsies show eosinophilic infiltration. This study defines the clinical and histological features of patients with eosinophilic esophagitis, distinguishing it from gastroesophageal reflux disease. Esophageal biopsies from patients with dysphagia or esophagitis were reviewed blindly, and assessed for: epithelial eosinophil counts, presence of eosinophilic microabscesses, edema, basal zone hyperplasia, lamina propria papillae elongation, eosinophils and fibrosis. Clinical and endoscopic findings were obtained. Eosinophilic esophagitis was diagnosed with epithelial eosinophils $\geq 15$ in $\geq 2$ high-power fields (hpfs) or $\geq 25$ in any hpf. Analysis was performed with Mann-Whitney, $\chi^{2}$ and ANOVA tests. Of 157 cases, 41 had eosinophilic esophagitis. Male gender $(81 \%)$ and age $\leq 45(54 \%)$ were commoner in patients with eosinophilic esophagitis $(P=0.001,0.010$, respectively). Dysphagia was more common in eosinophilic esophagitis patients (63\%, $\boldsymbol{P}<0.001)$; heartburn was more common in noneosinophilic esophagitis patients $(53 \%, P<0.001)$. Endoscopic rings were more common in eosinophilic esophagitis patients $(27 \%, P=0.023)$; hiatus hernia was more common in noneosinophilic esophagitis patients $(11 \%, P=0.022)$. Eosinophils were more numerous in eosinophilic esophagitis biopsies (mean $39 / \mathrm{hpf}, \boldsymbol{P} \leq \mathbf{0 . 0 0 1}$ ). Only eosinophilic esophagitis biopsies had eosinophilic microabscesses $(42 \%, P \leq 0.001)$. Edema, basal zone hyperplasia, lamina propria papillae elongation and lamina propria eosinophils were commoner in eosinophilic esophagitis $(P=<0.001-0.002)$, while lamina propria fibrosis was specific for eosinophilic esophagitis $(39 \%, P<0.001)$. Eosinophilic esophagitis is a disease with a predilection for young males with dysphagia and rings on endoscopy. Biopsies in eosinophilic esophagitis have high epithelial eosinophil counts, averaging nearly $40 / \mathrm{hpf}$. Increased awareness of eosinophilic esophagitis is necessary, since treatment with allergen elimination or antiinflammatory therapy may be more effective than acid suppression.
\end{abstract}

Modern Pathology (2006) 19, 90-96. doi:10.1038/modpathol.3800498; published online 7 October 2005

Keywords: eosinophil; eosinophilic; esophagitis

Eosinophilic esophagitis is well documented in children, but the adult form has only recently gained recognition as a distinct entity. Eosinophilic esophagitis was first described in adults by $\mathrm{Lee}^{1}$ in 1985, and occurs most often in young males with dysphagia and allergies, in whom esophageal biop-

Correspondence: Dr DK Driman, MBChB, FRCPC, Department of Pathology, London Health Sciences Centre and University of Western Ontario, 339 Windermere Road, London, Ontario, Canada N6A 5A5.

E-mail: ddriman@uwo.ca

Received 14 June 2005; revised 18 August 2005; accepted 26 August 2005; published online 7 October 2005 sies show eosinophil infiltration. Primary eosinophilic esophagitis can be divided into atopic, nonatopic and familial types; most patients have atopic sensitization to aeroallergens or food antigens, while the familial variant $(10 \%)$ is inherited in an autosomal dominant manner. ${ }^{2}$

Eosinophil infiltration of the esophagus is the cardinal pathological finding, albeit a nonspecific abnormality that occurs secondary to several unrelated diseases. Eosinophilic esophagitis is associated with primary systemic disorders of tissue eosinophilia, such as hypereosinophilic syndrome and eosinophilic gastroenteritis. However, esophageal eosinophil infiltration may also occur as a 
secondary phenomenon, with drug exposure, parasitic and fungal infections, gastroesophageal reflux disease, esophageal leiomyomatosis and scleroderma. ${ }^{2,3}$

Attwood et $a l^{4}$ were first to provide comparisons of eosinophilic esophagitis and gastroesophageal reflux disease, the latter being the most common cause of adult esophagitis. It is important to distinguish eosinophilic esophagitis from reflux esophagitis, not only because treatment differs but also because patients may suffer needless exposure to therapeutic maneuvers used for reflux esophagitis, such as long-term medical therapy with proton pump inhibitors, and invasive surgical procedures like fundoplication. This study distinguishes primary eosinophilic esophagitis from gastroesophageal reflux disease in an adult cohort, based on histological features correlated with epidemiological, clinical and endoscopic findings.

\section{Materials and methods}

All esophageal biopsies from patients with dysphagia or esophagitis from June 2002 to December 2004 were retrieved from the Pathology files at the London Health Sciences Centre (LHSC). The biopsies were reviewed by JRP and DKD, blinded to clinical information and previous pathological diagnoses. Biopsies for Barrett's esophagus or mass lesions were excluded. The number of sampled tissue fragments per biopsy was recorded and the following histological features were assessed: eosinophil counts in up to 10 high-power fields (hpfs) $\left(1 \mathrm{hpf}=0.44 \mathrm{~mm}^{2}\right)$; and presence or absence of intercellular edema, basal zone hyperplasia $>20 \%$ of the epithelial thickness, lamina propria papillae elongation to $>\frac{2}{3}$ of the epithelial height, lamina propria eosinophils, lamina propria fibrosis, neutrophils and eosinophilic microabscesses; the latter were diagnosed with aggregates of $\geq 4$ eosinophils. Eosinophils were counted where they appeared most populous in the biopsy.

Clinic and hospital charts were blindly reviewed by JRP, HAJ and DKD, with the aid of attending physicians, to obtain clinical information and endoscopic findings. Historical details recorded include principal presenting complaint, allergy history (asthma, atopy, other), treatment and response to treatment. Endoscopic findings recorded include inflammation, erosions, ulcerations, strictures, rings and any other notable features. Peripheral eosinophil counts were sought from clinical charts as well as the electronic patient information system used at LHSC; counts $>400 / \mathrm{mm}^{3}$ are considered elevated. Cases without available clinical information were excluded from the study. This process yielded 157 study cases.

Eosinophilic esophagitis was diagnosed with intraepithelial eosinophils $\geq 15$ in $\geq 2 \mathrm{hpfs}$ or $\geq 25$ in any single hpf. Recognizing that absolute cutoff criteria for the number of intraepithelial eosinophils required for diagnosing eosinophilic esophagitis is presently lacking, these criteria were decided upon by reviewing the existing literature on eosinophilic esophagitis and attempting to incorporate both findings of previous histopathological analyses and expert opinion (Table 1). Cases of histological eosinophilic esophagitis were then re-reviewed by JRP and DKD to further assess eosinophil distribution, degranulation and presence or absence of ulceration. Eosinophil distribution was recorded as superficial, peripapillary or diffuse, and eosinophil degranulation was graded as absent, minimal, moderate and severe. The percentage of sampled tissue involved by diagnostic numbers of eosinophils was also recorded. For all cases of histological eosinophilic esophagitis, accompanying gastric and duodenal biopsies were reviewed to exclude eosinophilic gastroenteritis. Statistical analysis was performed using $\chi^{2}$, Mann-Whitney and ANOVA tests in order to compare eosinophilic esophagitis patients with noneosinophilic esophagitis patients. Statistical significance was determined with an alpha level $P$-value of $<0.05$.

\section{Results}

\section{Clinical Features}

Using the above diagnostic criteria, 41 subjects with histological eosinophilic esophagitis were identified. The demographic features and clinical symptoms in eosinophilic esophagitis patients and noneosinophilic esophagitis patients are summarized in Table 2. The mean age of eosinophilic esophagitis patients was 45 years, who were, on average, 10 years younger than noneosinophilic esophagitis patients (mean age 55 years, $P=0.001$ ). Male gender (33 $(81 \%), P=0.001)$ and age $\leq 45$ years $(22(54 \%), P=0.010)$ were more common in patients with eosinophilic esophagitis. The male to female ratio among eosinophilic esophagitis patients was $4: 1$, while there was an equal number of males and females in the noneosinophilic esophagitis group. Dysphagia was more common in eosinophilic esophagitis patients (26 (63\%),

Table 1 Average number of eosinophils previously described in eosinophilic esophagitis

\begin{tabular}{|c|c|c|c|}
\hline Year & Author(s) & Publication type & Eosinophils/hpf \\
\hline 1985 & $\mathrm{Lee}^{1}$ & Original research & 10 \\
\hline 1993 & Attwood et $a l^{4}$ & Original research & 20 \\
\hline 1999 & Walsh et $a l^{7}$ & Original research & 31 \\
\hline 2000 & Orenstein et $a l^{\mathrm{B}}$ & Original research & 5 \\
\hline 2002 & Fox et $a l^{5}$ & Review & $15-20$ \\
\hline 2003 & Straumann et $a l^{10,12}$ & Original research & 25 \\
\hline 2003 & Croese et $a l^{11}$ & Original research & 30 \\
\hline 2003 & Liacouras $^{13}$ & Review & 20 \\
\hline 2004 & Potter et $a l^{9}$ & Original research & 15 \\
\hline 2004 & Rothenberg ${ }^{2}$ & Review & $7-24$ \\
\hline
\end{tabular}


$P<0.001$ ), while heartburn was more common in noneosinophilic esophagitis patients (62 (53\%), $P<0.001$ ); we regard the latter group to represent patients with gastroesophageal reflux disease. Asthma (6 $(15 \%), P<0.05)$ was more common in eosinophilic esophagitis patients compared to patients with gastroesophageal reflux disease (6 (5\%), $P<0.05)$.

The endoscopic findings in eosinophilic esophagitis and noneosinophilic esophagitis patients are summarized in Table 3. Patients with eosinophilic esophagitis were more likely to have abnormal endoscopic findings than noneosinophilic esophagitis patients. Endoscopic rings were more common in eosinophilic esophagitis (27 vs $11 \%, P=0.023$ ), while hiatus hernia was more common in patients with gastroesophageal reflux disease (11 vs $0 \%$, $P=0.022$ ). Rings were especially associated with eosinophilic esophagitis, being present more than twice as often in eosinophilic esophagitis as compared to patients with gastroesophageal reflux disease. While mucosal erosions or ulcers were less commonly seen among eosinophilic esophagitis patients, this difference was not statistically significant. The vast majority of biopsies were taken from the distal esophagus; only three of 35 were taken

Table 2 Demographics and symptoms of patients with eosinophilic esophagitis and gastroesophageal reflux disease

\begin{tabular}{|c|c|c|c|}
\hline & $\begin{array}{c}\text { Eosinophilic } \\
\text { esophagitis } \\
\text { patients }(\mathrm{n}=41) \\
\text { Mean } \pm \text { s.d. }\end{array}$ & $\begin{array}{c}\text { Noneosinophilic } \\
\text { esophagitis } \\
\text { patients }(\mathrm{n}=116) \\
\text { Mean } \pm \text { s.d. }\end{array}$ & $\mathrm{P}$-value \\
\hline \multirow[t]{2}{*}{ Age (years) } & $45 \pm 16$ & $55 \pm 15$ & 0.001 \\
\hline & n $(\%)$ & n $(\%)$ & $\mathrm{P}$-value \\
\hline Sex (M) & $33(81)$ & $59(51)$ & 0.001 \\
\hline Age $\leq 45$ (years) & $22(54)$ & $36(31)$ & 0.010 \\
\hline Dysphagia & $26(63)$ & $33(28)$ & $<0.001$ \\
\hline Heartburn & $8(20)$ & $62(53)$ & $<0.001$ \\
\hline Asthma & $6(15)$ & $6(5)$ & $<0.05$ \\
\hline
\end{tabular}

from either the mid or proximal esophagus. Of the 10 eosinophilic esophagitis patients with available peripheral eosinophil counts, only one patient had an elevated count of $800 / \mathrm{mm}^{3}$. The vast majority of patients in either group had been treated with proton pump inhibitors prior to biopsy. Among eosinophilic esophagitis patients, 93\% (37 of 40) were on PPIs, with a favorable response in only 15\% (6 of 40) of cases; only two eosinophilic esophagitis patients were treated with steroids, both with good response.

\section{Pathological Features}

The histological features of biopsies from eosinophilic esophagitis patients and noneosinophilic esophagitis patients are summarized in Table 4 . The average number of tissue fragments per biopsy available for assessment was three for both eosinophilic esophagitis and noneosinophilic esophagitis groups. Eosinophils were more numerous in biopsies from eosinophilic esophagitis patients (mean $39 / \mathrm{hpf}$ ) compared to patients with gastroesophageal reflux disease (mean 1/hpf, $P \leq 0.001$ ). Among biopsies from eosinophilic esophagitis patients, the distribution of eosinophils was often patchy; in $34 \%$ of these cases, $<50 \%$ of the sampled tissue showed diagnostic numbers of eosinophils. Lamina propria papillae elongation, basal zone hyperplasia, inter-

Table 3 Endoscopic findings in patients with eosinophilic esophagitis and gastroesophageal reflux disease

\begin{tabular}{lccc}
\hline & $\begin{array}{c}\text { Eosinophilic } \\
\text { esophagitis } \\
\text { patients (n=41) } \\
\mathrm{n}(\%)\end{array}$ & $\begin{array}{c}\text { Noneosinophilic } \\
\text { esophagitis } \\
\text { patients (n=116) } \\
\mathrm{n}(\%)\end{array}$ & P-value \\
\hline Normal & $5(12)$ & $33(28)$ & 0.037 \\
Stricture & $11(27)$ & $20(17)$ & $\geq 0.1$ \\
Ring & $11(27)$ & $13(11)$ & 0.023 \\
Ulcer & $5(12)$ & $21(18)$ & $\geq 0.1$ \\
Hiatal hernia & $0(0)$ & $13(11)$ & 0.022 \\
Erosion & $2(5)$ & $12(10)$ & $\geq 0.1$ \\
Esophagitis & $12(29)$ & $35(30)$ & $\geq 0.1$ \\
\hline
\end{tabular}

Table 4 Pathological features in patients with eosinophilic esophagitis and gastroesophageal reflux disease

\begin{tabular}{|c|c|c|c|}
\hline & $\begin{array}{c}\text { Eosinophilic esophagitis } \\
\text { patients }(\mathrm{n}=41) \\
\text { Mean (range) }\end{array}$ & $\begin{array}{c}\text { Noneosinophilic esophagitis } \\
\text { patients }(\mathrm{n}=116) \\
\text { Mean (range) }\end{array}$ & $\mathrm{P}$-value \\
\hline \multirow[t]{2}{*}{ Eosinophils/hpf } & $39(7-125)$ & $1(0-13)$ & $<0.001$ \\
\hline & $\mathrm{n}(\%)$ & $\mathrm{n}(\%)$ & $\mathrm{P}$-value \\
\hline Eosinophil microabscesses & $17(42)$ & $0(0)$ & $<0.001$ \\
\hline Increased lamina propria papillae & $30(73)$ & $32(28)$ & $<0.001$ \\
\hline Basal zone hyperplasia & $38(93)$ & $58(50)$ & $<0.001$ \\
\hline Edema & $39(95)$ & $58(50)$ & $<0.001$ \\
\hline Neutrophils & $8(20)$ & $38(33)$ & $\geq 0.1$ \\
\hline Lamina propria eosinophils & $23(56)$ & $48(41)$ & 0.002 \\
\hline Lamina propria fibrosis & $16(39)$ & $8(7)$ & $<0.001$ \\
\hline
\end{tabular}


cellular edema and lamina propria eosinophils were more common in eosinophilic esophagitis compared with gastroesophageal reflux disease $(73,93,95$ and $56 \%$ vs $28,50,50$ and $41 \%$; $P \leq 0.001-0.002$ ); while these features were not uncommonly seen in biopsies from patients with gastroesophageal reflux disease, the changes were more severe in eosinophilic esophagitis cases. Among biopsies from patients with eosinophilic esophagitis, lamina propria eosinophils and papillae elongation could not be evaluated in 24 and $15 \%$ of cases, respectively, as some biopsies were either poorly oriented or lacked lamina propria.

Eosinophilic microabscesses and lamina propria fibrosis, while present in only 42 and $39 \%$ of biopsies from eosinophilic esophagitis patients, respectively, were relatively specific for eosinophilic esophagitis $(P<0.001)$. There was sufficient lamina propria to evaluate in $76 \%$ of the biopsies from the eosinophilic esophagitis group. Ulceration was rare in biopsies from eosinophilic esophagitis patients, seen in only one case. Among eosinophilic esophagitis patients, the distribution of eosinophils was peripapillary in $41 \%$, diffuse in $34 \%$ and superficial in $27 \%$. In biopsies where eosinophils were present diffusely, they were also present around papillae, suggesting that peripapillary eosinophils may represent an earlier stage of epithelial infiltration. When eosinophils were present superficially, biopsies typically had a 'moth-eaten' appearance due to marked intercellular edema and associated acantholysis; eosinophilic microabscesses were often present at the luminal edge of the epithelium in such cases. Eosinophil degranulation was moderate to severe in $61 \%$ of biopsies from eosinophilic esophagitis patients. The degree of degranulation seemed to correlate with the number of intraepithelial eosinophils. Figures $1-3$ show the

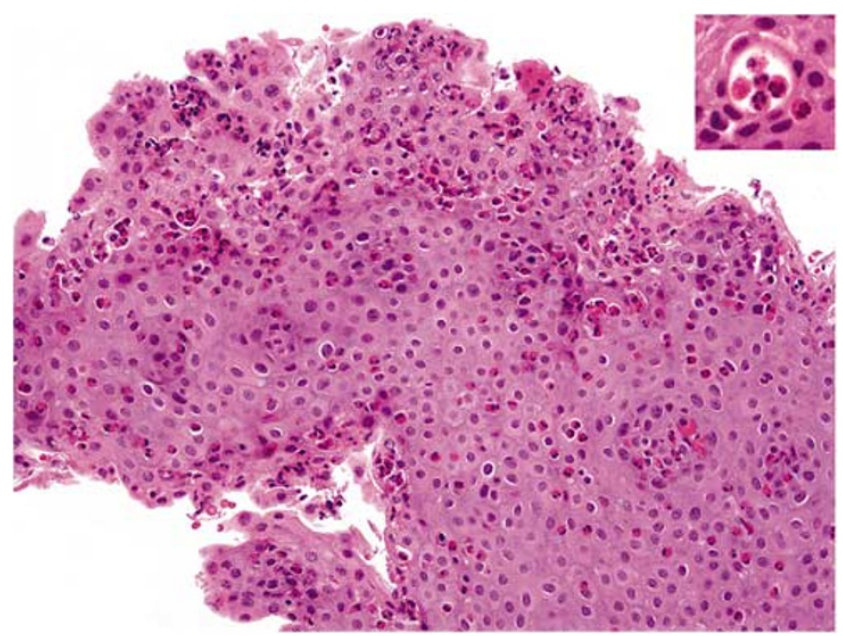

Figure 1 There is a large number of intraepithelial eosinophils present mainly in a superficial distribution. The inset demonstrates one of several eosinophilic microabscesses in this biopsy of an eosinophilic esophagitis patient (hematoxylin-eosin, original magnification $\times 250$; inset $\times 400$ ). typical histological features seen in our eosinophilic esophagitis patients and Table 5 provides a summary list of the pathology of eosinophilic esophagitis. Review of gastric and duodenal biopsies from eosinophilic esophagitis patients did not show increased numbers of eosinophils.

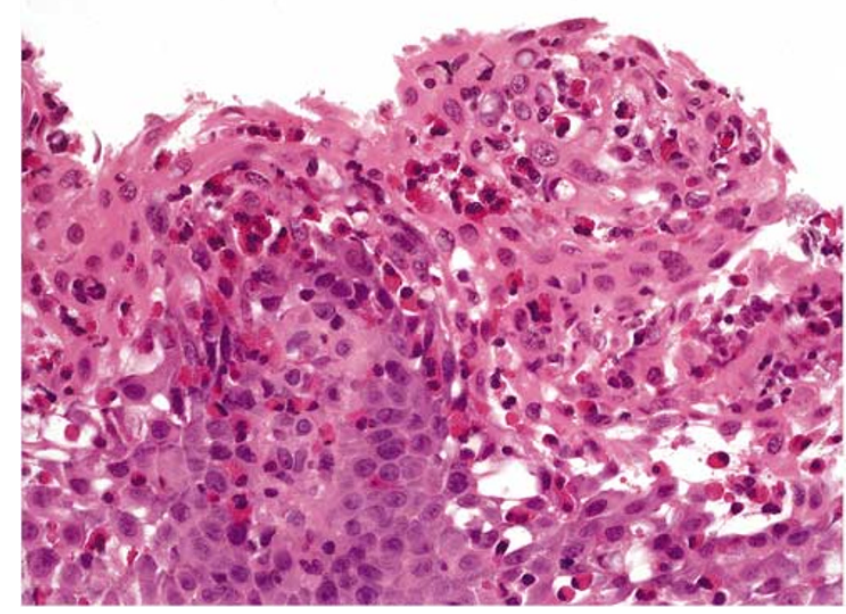

Figure 2 Many eosinophils are present at the luminal surface in this biopsy of an eosinophilic esophagitis patient. Notice the eosinophilic microabscesses and 'moth-eaten' appearance due to intercellular edema (hematoxylin-eosin, original magnification $\times 400)$.

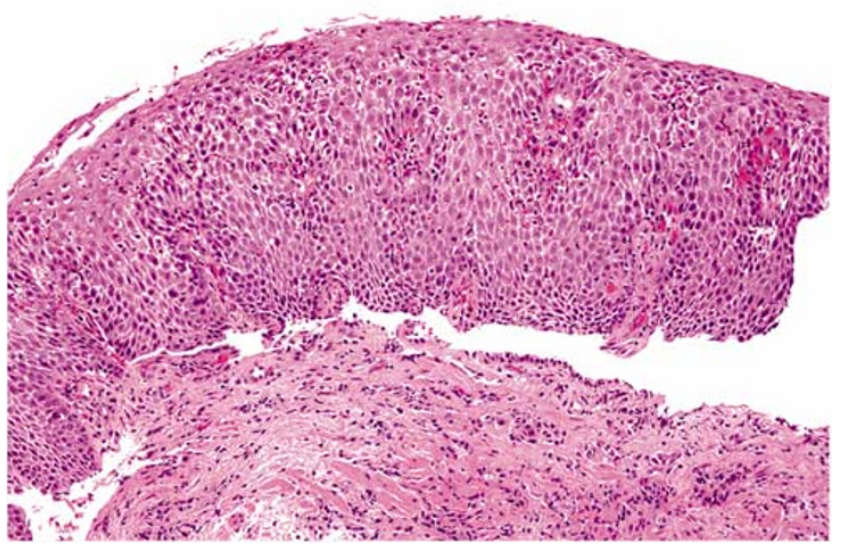

Figure 3 Lamina propria papillae elongation, basal zone hyperplasia, intercellular edema and lamina propria fibrosis are all evident in this well-oriented biopsy of an eosinophilic esophagitis patient (hematoxylin-eosin, original magnification $\times 100)$.

Table 5 Pathological features of eosinophilic esophagitis

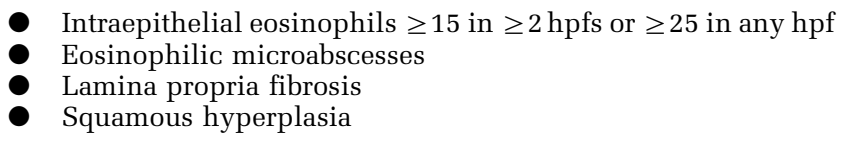




\section{Discussion}

While gastrointestinal disorders with eosinophil infiltration have been increasing over the past decade, the pediatric literature has reported a miniepidemic of eosinophilic esophagitis in particular. $^{2}$ Although the population incidence and prevalence of eosinophilic esophagitis have not been definitively studied, pediatric studies have estimated rates of up to $6 \%$ among patients with esophagitis. ${ }^{2}$ While $26 \%$ of our study patients had eosinophilic esophagitis histologically, this likely overestimates the true incidence of eosinophilic esophagitis in southwestern Ontario. As our case selection criteria was limited to patients with either dysphagia or esophagitis, some patients with either dyspepsia or gastroesophageal reflux may have been excluded; thus, patients with eosinophilic esophagitis, who typically present with dysphagia, are likely over-represented in our study group. Most eosinophilic esophagitis studies report a male predominance, including $>75 \%$ of reported adult and child cases. ${ }^{5}$ In adults, diagnosis typically occurs in the 3rd to 4th decade, but symptoms are often long standing by the time of diagnosis. In our eosinophilic esophagitis study population, the ratio of males to females was 4:1 and the average age at diagnosis was 45 years. Thus, male sex and younger age are strong features of eosinophilic esophagitis; however, females and the elderly are also susceptible to this disease. ${ }^{5}$

As demonstrated in our study, the most characteristic symptom among adults with eosinophilic esophagitis is dysphagia. In contrast to gastroesophageal reflux disease, food impaction is often the first symptom patients report. ${ }^{5}$ Esophageal manometry often demonstrates tertiary and simultaneous contractions and diffuse spasm, which correlates with impaction episodes in ambulatory studies. ${ }^{5,6}$ While such esophageal dysmotility is typical, a subset of patients do have strictures to explain their dysphagia. While adults may have a minor degree of concomitant heartburn, children with eosinophilic esophagitis characteristically present with gastroesophageal reflux disease-like symptoms, as well as nausea, vomiting, abdominal pain, feeding refusal and poor weight gain..$^{5,7,8}$ Among adults with eosinophilic esophagitis, studies report personal or family histories of allergies ranging from 50 to $90 \%$, including up to $60 \%$ with asthma and up to $25 \%$ with food allergies. ${ }^{9-11} \mathrm{~A}$ disproportionate number of our eosinophilic esophagitis patients also had asthma (at least 15\%); our study may underestimate the prevalence of asthma among eosinophilic esophagitis patients, as specific reporting of allergy histories was often omitted from the clinical record.

While endoscopic findings may be subtle and not infrequently missed, most patients do have gross abnormalities, including granularity, vertical furrowing, corrugation, concentric rings or stric- tures. ${ }^{5,9,11}$ Recently, Straumann et al have suggested that fragile esophageal mucosa, so-called 'crepe paper', which tears easily with minor trauma, may be pathognomic for eosinophilic esophagitis. ${ }^{12}$ In our study, rings were more commonly reported in eosinophilic esophagitis patients vs patients with gastroesophageal reflux disease; consequently, these findings likely represent intermittent circular muscle contraction rather than true congenital rings. Other findings reported to be characteristic of eosinophilic esophagitis include speckled whitish exudates and a small caliber esophagus. ${ }^{5}$ Some authors believe the former to represent superficial eosinophilic microabscesses, a marker of disease activity, and the latter to be a long segment esophageal stricture due to lamina propria fibrosis, a marker of disease chronicity. Although $27 \%$ of our study patients had superficially distributed eosinophils, often with associated eosinophilic microabscesses, white exudates were not described at the time of endoscopy. Other endoscopy studies have also suggested that exudates may be less frequently seen in adults, as compared to children, with eosinophilic esophagitis. ${ }^{9,11}$ Further studies correlating the presence of white exudates with eosinophilic microabscesses and superficially distributed eosinophils in adults with eosinophilic esophagitis are necessary to clarify the relationship between these endoscopic and histological features. As our study demonstrates, esophageal changes in eosinophilic esophagitis appear to occur in the absence of mucosal erosion or ulceration, in contradistinction to gastroesophageal reflux disease.

A strict consensus regarding the number of eosinophils within esophageal squamous epithelium required to make a diagnosis of eosinophilic esophagitis is lacking. While many studies have used >15-20 eosinophils/hpf as a diagnostic cutoff, others have used thresholds as low as $>5 / \mathrm{hpf}$ and as high as $>30 / \mathrm{hpf}$ (Table 1).$^{5,8-11,13}$ Rothenberg et $a 1^{2,14}$ suggest that eosinophil counts $<7 / \mathrm{hpf}, 7-24 / \mathrm{hpf}$ and $>24 / \mathrm{hpf}$ indicate gastroesophageal reflux disease, gastroesophageal reflux disease combined with allergy and primary eosinophilic esophagitis respectively. Although eosinophilic esophagitis patients in our study had an average of nearly 40 eosinophils/ hpf, many biopsies showed a patchy distribution and multiple hpfs within a single biopsy often lacked eosinophils. After considering previously published histopathological analyses and expert opinion, we consider intraepithelial eosinophils $\geq 15$ in $\geq 2 \mathrm{hpfs}$ or $\geq 25$ in any hpf to be reasonable and practically useful diagnostic criteria for histological eosinophilic esophagitis. Of course, primary eosinophilic esophagitis is a clinicopathological diagnosis to be made only when the secondary causes have been excluded. Involvement of the proximal esophagus is helpful in distinguishing eosinophilic esophagitis from gastroesophageal reflux disease, where eosinophils are typically confined to the distal end, but the mid-esophagus may 
lack eosinophils in up to $80 \%$ of eosinophilic esophagitis patients. ${ }^{13}$ We do not require biopsies of the proximal esophagus for the diagnosis of eosinophilic esophagitis, because in the vast majority of cases, endoscopists routinely biopsy the distal esophagus in the process of excluding gastroesophageal reflux disease or Barrett's esophagus; use of the criteria that we describe enables a diagnosis of eosinophilic esophagitis to be made on distal esophageal biopsies. Nevertheless, when endoscopists entertain the differential diagnosis of eosinophilic esophagitis, biopsies should be taken from the proximal and distal esophagus, as well as the gastric antrum and duodenum, the latter for the purpose of excluding eosinophilic gastroenteritis. Further, in a third of biopsies from our eosinophilic esophagitis patients, less than half of the sampled tissue showed diagnostic numbers of eosinophils, highlighting the patchiness of the disease and the need for multiple biopsies in order to maximize diagnostic yield.

Eosinophils are generally physiologic in the GI tract; their principal function includes provision of innate immunity against helminthic infections, although some authors have suggested that they may also play a role in organ development. ${ }^{2}$ The presence of eosinophils in Peyer's patches or intraepithelial locations are characteristic of gastrointestinal disorders with eosinophil infiltration; importantly, any number of eosinophils in the esophagus indicates pathology. ${ }^{2}$ Eosinophils have several toxic effects, primarily related to release of their secondary granules. In addition to epithelial damage, released major basic protein antagonizes muscarinic M2 receptors, causing vagal dysregulation and increased smooth muscle reactivity, likely contributing to dysphagia-type symptoms., ${ }^{2,15}$ This provides the rationale for including degranulating eosinophils and assessing the degree of degranulation in our study.

Straumann et $a l^{15}$ have demonstrated increased numbers of intraepithelial T cells (nearly ubiquitous in biopsies of patients with esophagitis) and mast cells (inconspicuous on routine H\&E) in esophageal biopsies of patients with eosinophilic esophagitis. In their study, $\mathrm{T}$ cells overexpressed IL-5, an eosinophil growth and activating factor produced by $\mathrm{TH} 2$ cells and mast cells in chronic allergic reactions. These findings suggest that eosinophilic esophagitis may be mechanistically related to asthma, since both diseases show CD4 TH2 cellIL-5-mast cell-dependent pathways leading to a chronic allergic-type inflammatory condition. As mast cell degranulation and release of histamine and leukotriene $\mathrm{C} 4$ stimulate smooth muscle contraction, eosinophils and mast cells may work in concert to produce the esophageal spasms, which cause dysphagia, a phenomenon reminiscent of the bronchoconstriction seen in asthmatic patients. ${ }^{15}$

Several authors have suggested an intimate relationship between eosinophilic esophagitis and asthma and studies of murine models have shown that repeated exposure of the lungs to aeroallergens such as Aspergillus fumigatus or the TH2 cytokine IL-13 induces eosinophilic esophagitis., ${ }^{2,16,17}$ Rothenberg et al have therefore suggested that development of eosinophilic esophagitis follows sensitization to aeroallergens in the respiratory tract, with subsequent swallowing of these aeroallergens leading to a hypersensitivity response and esophageal eosinophil infiltration. ${ }^{14}$ While both eosinophilic esophagitis and asthma have been increasing in prevalence over the past decade in young males, who often report seasonal variation of symptoms, definitive epidemiological data linking the two diseases is lacking. ${ }^{18}$

One should always consider the possibility of hypereosinophilic syndrome in a patient with a gastrointestinal disorder with eosinophil infiltration, especially when extra-GI manifestations, such as splenomegaly, cutaneous, cardiac or respiratory findings are present. ${ }^{2}$ Patients with eosinophilic esophagitis and elevated blood eosinophil counts $>1500 / \mathrm{mm}^{3}$ meet the criteria for hypereosinophilic syndrome. ${ }^{2}$ While most studies suggest that peripheral blood eosinophilia is uncommon with eosinophilic esophagitis (in contradistinction to eosinophilic gastroenteritis), the consequence of a missed diagnosis of hypereosinophilic syndrome could be serious, as cardiac involvement can be life threatening, and patients need to be monitored with routine echocardiograms. ${ }^{2}$

Presently, the mainstay of therapy for patients with eosinophilic esophagitis is dietary modification. ${ }^{13,19}$ While a trial of food and aeroallergen avoidance is indicated for patients with eosinophilic esophagitis, determination of specific allergens can be difficult, as skin and radioallergosorbent (RAST) testing for IgE is often negative. ${ }^{13,19} \mathrm{~A}$ diet composed of an elemental formula may bring clinical and histological improvement or resolution in patients with disease resistant to elimination of specific allergens. ${ }^{13,19}$ Alternatively, steroids, either systemic or topical, may achieve satisfactory results. Use of topical products like fluticasone dipropionate avoids the protean side effects of systemic steroids, undergoing first-pass metabolism in the liver after GI absorption. ${ }^{13,19,20}$ Eosinophilic esophagitis complicated by strictures can be treated with dilators, but response durability is uncertain, the procedure may be painful and the risk of tearing or perforation requires endoscopists to proceed down such a therapeutic path only with great caution. ${ }^{5}$ While acid suppression therapy may improve symptoms, histological improvement does not generally occur. ${ }^{5,13}$ Importantly, surgical fundoplication has not proven to be effective and patients should be spared this invasive procedure. ${ }^{5,13}$

In summary, eosinophilic esophagitis in adults is a distinct clinicopathological disease with a predilection for younger males with dysphagia and rings on endoscopy. Pathologists should be aware that esophageal biopsies with high intraepithelial 
eosinophil counts, particulary in younger males with dysphagia, likely represents eosinophilic esophagitis. This is of clinical importance, since allergen elimination or anti-inflammatory therapy may be more effective than acid suppression in these patients.

\section{References}

1 Lee R. Marked eosinophilia in esophageal mucosal biopsies. Am J Surg Pathol 1985;9:475-479.

2 Rothenberg ME. Eosinophilic gastrointestinal disorders (EGID). J Allergy Clin Immunol 2004;113:11-28.

3 Mansoor A, Soetikno R, Ahmed A. The differential diagnosis of eosinophilic esophagitis. J Clin Gastroenterol 2000;30:242-244.

4 Attwood SEA, Smyrk TC, Demeester TR, et al. Esophageal eosinophilia with dysphagia. A distinct clinicopathological syndrome. Dig Dis Sci 1993;38: 109-116.

5 Fox VL, Nurko S, Furuta GT. Eosinophilic esophagitis: it's not just kid's stuff. Gastrointest Endosc 2002; 56:260-270.

6 Nurko S, Fox V, Fortunato C, et al. Esophageal motor abnormalities in patients with allergic esophagitis. A study with prolonged esophageal $\mathrm{pH} /$ manometry [abstract]. J Pediatr Gastroenterol Nutr 2001;33:417.

7 Walsh SV, Antonioli DA, Goldman H, et al. Allergic esophagitis in children: a clinicopathological entity. Am J Surg Pathol 1999;23:390-396.

8 Orenstein SR, Shalaby TM, Di Lorenzo C, et al. The spectrum of pediatric eosinophilic esophagitis beyond infancy: a clinical series of 30 children. Am J Gastroenterol 2000;95:1422-1430.

9 Potter JW, Saeian K, Staff D, et al. Eosinophilic esophagitis in adults: an emerging problem with unique esophageal features. Gastrointest Endosc 2004; 59:355-361.

10 Straumann A, Spichtin HP, Grize L, et al. Natural history of primary eosinophilic esophagitis: a followup of 30 adult patients for up to 11.5 years. Gastroenterology 2003;125:1660-1669.

11 Croese J, Fairley SK, Masson JW, et al. Clinical and endoscopic features of eosinophilic esophagitis in adults. Gastrointest Endosc 2003;58:516-522.

12 Straumann A, Rossi L, Simon HU, et al. Fragility of the esophageal mucosa: a pathognomonic endoscopic sign of primary eosinophilic esophagitis? Gastrointest Endosc 2003;57:407-412.

13 Liacouras CA. Eosinophilic esophagitis in children and adults. J Pediatr Gastroenterol Nutr 2003;37 (Suppl):S23-S28.

14 Rothenberg ME, Mishra A, Collins MH, et al. Pathogenesis and clinical features of eosinophilic esophagitis. J Allergy Clin Immunol 2001;108:891-894.

15 Straumann A, Bauer M, Fischer B, et al. Idiopathic eosinophilic esophagitis is associated with a TH2type allergic inflammatory response. J Allergy Clin Immunol 2001;108:954-961.

16 Mishra A, Hogan SP, Brandt EB, et al. An etiological role for aeroallergens and eosinophils in experimental esophagitis. J Clin Invest 2001;107:83-90.

17 Mishra A, Rothenberg ME. Intratracheal IL-13 induces eosinophilic esophagitis by an IL-5, eotaxin-1, and STAT6-dependent mechanism. Gastroenterology 2003; 125:1419-1427.

18 Hartert TV, Peebles Jr RS. Epidemiology of asthma: the year in review. Curr Opin Pulm Med 2000;6:4-9.

19 Markowitz JE, Liacouras CA. Eosinophilic esophagitis. Gastroenterol Clin N Am 2003;32:949-966.

20 Teitelbaum JE, Fox VL, Twarog FJ, et al. Eosinophilic esophagitis in children: immunopathological analysis and response to fluticasone propionate. Gastroenterology 2002;122:1216-1225. 